\title{
Impact of Smoking and Tobacco Use on the Severity of COVID-19 Infection
}

\author{
Zeina Aoun-Bacha and Moussa Riachy \\ Department of Pulmonary and Critical Care Medicine, Hotel Dieu de France Medical Center (HDFMC) of the Saint-Joseph University (USJ), \\ Beirut, Lebanon
}

DOI: https://doi.org/10.17925/USPRD.2020.5.1.12

S moking, in all forms, such as cigarettes, waterpipe, and e-cigarettes, is harmful and proven to cause lung injury. In this pandemic era, it is essential to examine potential evidence linking smoking and the risk of infection with severe acute respiratory syndrome coronavirus 2 (SARS-CoV-2), as well as the prognosis and severity of COVID-19 disease. The effect of smoking and nicotine on airway inflammation has been established in multiple studies. However, the impact of nicotine on the angiotensin-converting enzyme 2 receptor remains controversial and calls for more studies.

\section{Keywords}

Smoking, nicotine, COVID-19

Disclosures: Zeina Aoun and Moussa Riachy have no financial or non-financial relationships or activities to declare in relation to this article.

Review Process: Double-blind peer review.

Compliance with Ethics: This article is an opinion piece and does not report on new clinical data, or any studies with human or animal subjects performed by either of the authors.

Authorship: The named authors meet the Internationa Committee of Medical Journal Editors (ICMJE) criteria for authorship of this manuscript, take responsibility for the integrity of the work as a whole, and have given final approval for the version to be published.

Access: This article is freely accessible at touchRESPIRATORY.com (c) Touch Medical Media 2020.

Received: May 12, 2020

Accepted: June 14, 2020

Published Online: December 23, 2020

Citation: US Respiratory and Pulmonary Diseases. 2020;5(1):12-4

Corresponding Author: Zeina Aoun-Bacha, Hotel Dieu de France Medical Center (HDFMC) of

Saint-Joseph University (USJ), Alfred Naccache Boulevard, Achrafieh Area, 166830, Beirut, Lebanon

E: zeina.aoun@hdf.usj.edu.lb

ORCID ID: orcid.org/0000-0001-9723-1267

Support: No funding was received in the publication of this article.
Smoking is a significant risk factor for severe COVID-19. A substantial amount of evidence supports the fact that tobacco use and chronic obstructive pulmonary disease (COPD) are among the strongest risk factors that lead to severe disease and high mortality due to COVID-19 illness. In a recent publication, Zhao et al. carried out a meta-analysis and systemic review of published literature on the prevalence of COPD and smoking in severe hospitalized patients with COVID-19. The pooled odds ratio was found to be 4.38 for COPD and 1.98 for smoking. They concluded that COPD and current smoking are strong factors that worsen the progression and outcome of COVID-19 illness. ${ }^{1}$

The preliminary recommendations issued by the World Health Organization (WHO) stated that smokers are 14 times more likely to develop severe COVID-19. ${ }^{2}$ This statement was based on the results of a study published in the Chinese Medical Journal, conducted in a group of 78 individuals, where the odds ratio for the history of smoking regarding COVID-19 severity was 14.285 , the highest amongst the considered risk factors affecting the disease progression. ${ }^{3} \mathrm{~A}$ meta-analysis followed, confirming that smokers with COVID-19 are 2.5 times more likely to need mechanical ventilation and intensive care unit (ICU) admission, and have a significantly higher risk of mortality. ${ }^{4}$ Shortly after the COVID-19 outbreak, The Global Initiative for Chronic Obstructive Lung Disease (GOLD) issued recommendations in which they recognize that people with COPD are amongst the worst affected by COVID-19.5

We know from various studies that chronic respiratory disease is always present in patients with severe COVID-19, and is one of the established risk factors that are associated with worse outcomes, along with diabetes and cardiovascular diseases (including hypertension). ${ }^{6}$ As the number of comorbidities increases, so does the risk of severe disease. ${ }^{6}$ However, there are some studies that contradict this, and claim that smoking does not affect COVID-19 severity. Lippi and Henry reported that active smoking was not found to be a significant predictor of COVID-19 severity, despite the substantial trend towards higher risk. In addition to this, Vardavas and Nikitara reported that smoking is most likely associated with negative progression and adverse outcomes of COVID-19. ${ }^{4}$ Each of these systematic reviews evaluated five studies (some of which were common to both analyses), but the available data was not robust, and the analysis provided failed to adjust for other potential important factors, such as gender, comorbidities, and age. ${ }^{8}$ There are other meta-analyses based on more studies and more significant data, which confirm the impact of smoking in the severity of COVID-19. Sánchez et al. concluded that the results of the meta-analysis do not necessarily rule out the association between the use of tobacco products and COVID-19 severity. ${ }^{8}$

Smoking remains the most critical risk factor responsible for the major non-communicable diseases that cause most deaths worldwide. ${ }^{9}$ It is an established independent risk factor for ischemic heart disease, cerebrovascular disease (stroke), COPD, and lower respiratory infections (mainly pneumonia). ${ }^{\circ}$ In the pre-COVID-19 era, when smokers were compared with non-smokers, smokers showed chronic 
inflammation of the airways, lung tissue, and pulmonary blood vessels, caused by exposure to inhaled irritants such as tobacco smoke and other pollutants. ${ }^{10}$

One element that was extensively studied with regard to its impact on lung irritation is histone deacetylase 2 (HDAC2). HDAC2, being one of the class I histone deacetylases, is critical for regulating the expression of inflammatory genes. The effect of smoking and COPD on HDAC2 is described in many experimental studies. In their article, sohal et al. assessed the expression of HDAC2 in the airways of smokers and patients with COPD."1 They found that, in the case of smokers, HDAC2 expression is increased in the lamina propria. On the other hand, they found that it is reduced in patients with COPD, and becomes worse with severity of the disease. Ex-smokers have normalized HDAC2 cell expression..$^{1,12}$ Barnes, in his article, talks about the decrease of the HDAC2 enzyme in peripheral lung and alveolar macrophages, ${ }^{13}$ which is responsible for the inhibition of histone acetylation, a significant trigger for inflammation in the airways. This histone acetylation increases angiotensin-converting enzyme 2 (ACE2) expression and up-regulation in pulmonary diseases like COPD and pulmonary arterial hypertension when compared with controls. ${ }^{14}$

In addition to its correlation with the severity of the COVID-19 illness, smoking can increase the risk of contamination by severe acute respiratory syndrome coronavirus 2 (SARS-CoV-2). In fact, most, if not all devices used for smoking, require the individual to raise the device to their mouth, thereby touching their mouth with their hands. Thus, the simple act of raising the smoking device to the mouth forces the smokers to break the primary prevention recommended in order to avoid contamination, exposing the individual to self-contamination.

Waterpipe smoking is another tobacco-burning device, very popular in the Middle-East region, and is gaining popularity around the world. The usual nature of waterpipe smoking means that a single mouthpiece and hose are often shared between people, especially in social and communal settings. Thus, waterpipe use can increase the transmission of infectious diseases, respiratory, and otherwise, between users. This increased risk was confirmed with many infectious agents such as respiratory viruses, herpes simplex virus, and tuberculosis. ${ }^{2}$ Also, the waterpipe device itself may contribute to the risk of contamination by providing an environment that promotes the survival of microorganisms outside the body. This is primarily due to the moisture in tobacco smoke and the use of cold water in the water chamber for a cold airflow, which may facilitate the survival of viruses and bacteria. ${ }^{2}$

New-generation devices, such as IQOS (I Quit Ordinary Smoking) and e-cigarettes, are commonly construed by the media as "healthier" than regular cigarettes. In fact, they are advertised by cigarette companies as "reduced-risk products." However, this is misleading, and studies have shown that these modern devices are toxic to the cells. ${ }^{15-17}$ In fact, in these studies, the researchers compared the effect of these three nicotine sources on different types of cells taken from the human airways. They found that, although there was a difference in the levels of toxicity between the different nicotine-delivery products, they all damaged the cells and affected their normal functions. Regarding COVID-19, these modern devices also cause an additional risk. In fact, smoking IQOS or e-cigarettes tends to generate a substantial volume of vapor exhaled, which can lead to viral transmission by droplets. ${ }^{17}$
Human ACE2 protein, the most important known vector of the coronavirus, lies on the surface of lung cells and is responsible for triggering the inflammatory response. Multiple well-conducted studies have shown that patients with COPD and current smokers have a significantly increased expression of ACE2 in their lungs and airways. ${ }^{14,18}$ Since smokers and patients with COPD have overexpression of ACE2 in their lungs, they are predisposed to more severe COVID-19. Nicotine also has a direct impact on the recognized receptor for the virus (ACE2), leading to the inflammatory reaction of the lung's epithelial cells. ${ }^{2}$ This effect of nicotine is not yet fully understood due to the complexity of the renin-angiotensin system (RAS), and requires further investigation. Two studies, not yet peer-reviewed, published in the same month, offered contradictory hypotheses regarding the effect of nicotine. The study conducted in France assumes a protective effect of nicotine on the ACE2 receptor; ;12 they proposed that nicotinic acetylcholine receptors can play a role in SARS pathophysiology, and tried to justify their hypothesis. In contrast, the Jodhpur study by the Indian Institute of Technology, showed that nicotine can alter the homeostasis of RAS, contributing to the development of cardiovascular and pulmonary diseases. ${ }^{19}$

The risk of increasing inflammation induced by the e-cigarette is still unknown, ${ }^{20}$ and more focused studies are required to determine the adverse effects of e-cigarettes on patients with COVID-19. However, since vaping is a nicotine provider, it may also be an inducer of this inflammatory response in the airways. ${ }^{2}$ We all know about the nationwide outbreak of e-cigarette or vaping, product use-associated lung injury. ${ }^{21}$

The low prevalence of COVID-19 in patients with COPD and smokers was raised in studies from China and the USA. ${ }^{20}$ Only $12.6 \%$ of the people hospitalized for COVID-19 in China were smokers, and this rate was even lower in the USA (1.3\%). Guan et al. related these results, most probably, to under-diagnosis. ${ }^{\circ}$ Smoking status was only self-reported in the majority of the cases. The prevalence of current self-reported smoking in patients with a COVID-19 diagnosis can sometimes be less than 6.5\%, and could vary from 1.4-12.6\%, (95\% confidence interval 4.9-8.2\%) depending on the study. ${ }^{20}$ This was analyzed in detail by Cattaruzza et al., whereby they agreed that low prevalence among hospitalized patients is partially due to the misclassification of many smokers as non-smokers. For example, someone who quit smoking by the day of hospitalization, could be misclassified as "former smoker."22 Also, hospitalized patients are generally older, hence, more prone to having comorbidities, with a higher probability of them having been former smokers.

Changeux et al. propose the use of nicotine replacement therapy as a preventive measure in the coronavirus pandemic. ${ }^{12}$ However, at this stage, this cannot be justified. Nicotine is to be used only for smoking cessation treatment. Nicotine is a very highly addictive substance, associated with numerous side effects, and should not be initiated in non-smokers.

We emphasize the need for effective preventive measures to support reducing the burden of COVID-19 on smokers and patients with COPD. The pandemic may present an opportunity to motivate smokers further to quit smoking due to the risk of more severe disease. We cannot stress enough the importance of smoking cessation to patients with COPD and current smokers during this COVID-19 period. 
1. Zhao $Q$, Meng M, Kumar R, et al. The impact of COPD and smoking history on the severity of COVID-19: A systemic review and meta-analysis. J Med Virol. 2020;92:1915-21.

2. World Heath Organisation (WHO). Tobacco and waterpipe use increases the risk of COVID-19. 2020. Available at: www.emro.who.int/tfi/know-the-truth/tobacco-and-waterpipeusers-are-at-increased-risk-of-covid-19-infection.html (accessed August 14, 2020)

3. Liu W, Tao Z-W, Wang L, et al. Analysis of factors associated with disease outcomes in hospitalized patients with 2019 novel coronavirus disease. Chin Med J. 2020;133:1032-8.

4. Vardavas C, Nikitara K. COVID-19 and smoking: A systematic review of the evidence. Tob Induc Dis. 2020;18:20.

5. Global Initiative for Chronic Obstructive Lung Disease. GOLD COVID-19 Guidance. Available at: https://goldcopd.org/goldcovid-19-guidance/ (accessed June 18, 2020).

6. Guan W, Liang W, Zhao Y, et al. Comorbidity and its impact on 1590 patients with Covid-19 in China: A nationwide analysis. Eur Resp J. 2020;55:2000547.

7. Lippi G, Henry BM. Active smoking is not associated with severity of coronavirus disease 2019 (COVID-19). Eur J Intern Med.2020;75:107-8.

8. Sánchez J, Acevedo N, Guzmán E. Active smoking and severity of coronavirus disease 2019 (COVID-19): Differences in measurement of variables could cause errors in the results. Eur I Intern Med.
2020;77:127-8.

. Jha P, Peto R. Global effects of smoking, of quitting, and of taxing tobacco. N Eng J Med. 2014;37:60-8.

10. Centers for Disease Control and Prevention (US): National Center for Chronic Disease Prevention and Health Promotion (US); Office on Smoking and Health (US). How tobacco smoke causes disease: The biology and behavioral basis for smoking-attributable disease: A report of the Surgeon General. Atlanta (GA): Centers for Disease Control and Prevention (US); 2010. Available from: www.ncbi.nlm.nih.gov/books/NBK53017/ (accessed August 14, 2020)

11. Sohal SS, Reid D, Soltani A, et al. Changes in airway histone deacetylase 2 in smokers and COPD with inhaled corticosteroids: A randomized controlled trial. PLOS One. 2013:8:e64833.

12. Changeux J-P, Amoura Z, Rey F, Miyara M. A nicotinic hypothesis for Covid-19 with preventive and therapeutic implications. $C R$ Biol. 2020;343:33-9.

13. Barnes PJ. Role of HDAC2 in the pathophysiology of COPD. An Rev Physiol. 2009;71:451-64.

14. Pinto BG, Oliveira AE, Singh Y, et al. ACE2 expression is increased in the lungs of patients with comorbidities associated with severe COVID-19. J Infect Dis. 2020;222:556-63.

15. ScienceDaily. New heated tobacco device causes same damage to lung cells as e-cigs and smoking, study finds. February 11, 2019.
Available at: wnw.sciencedaily.com/releases/2019/02/190211083119.htm (accessed June 18, 2020)

16. Sohal SS, Eapen MS, Naidu VG, Sharma P. IQOS exposure impairs human airway cell homeostasis: Direct comparison with traditional cigarette and e-cigarette. ERJ Open Res. 2019;5:00159-2018.

17. MCAlinden KD, Eapen MS, Lu W, et al. COVID-19 and vaping: Risk for increased susceptibility to SARS-COV-2 infection? Eur Respir 」. 2020;56:2001645.

18. Leung JM, Yang CX, Tam A, et al. ACE-2 expression in the small airway epithelia of smokers and COPD patients: Implications for COVID-19. Eur Respir J. 2020;55:2000688.

19. Das G, Mukherjee N, Ghosh S. Neurological insights of COVID-19 pandemic. ACS Chem Neurosci. 2020;11:1206-9.

20. Farsalinos K, Barbouni A, Niaura R. Smoking, vaping and hospitalization for COVID-19. Qeios. 2020; doi:10.32388/z6908a.13

21. Krishnasamy VP, Hallowell BD, KO JY, et al. Update: Characteristic of a nationwide outbreak of e-cigarette, or vaping, product useassociated lung injury — United States, August 2019-January 2020 MMWR Morb Mortal Wkly Rep. 2020;69:90-4

22. Cattaruzza MS, Zagà V, Gallus S, et al. Tobacco smoking and COVID-19 pandemic: old and new issues. A summary of the evidence from the scientific literature. Acta Biomed. 2020:91:106-12. 